\title{
Design Of Lunar-Gravity-Assisted Escape Trajectories
}

\section{Lorenzo Casalino $^{1}$ (1) . Gregory Lantoine ${ }^{2}$}

Published online: 7 August 2020

(C) The Author(s) 2020

\begin{abstract}
Lunar gravity assist is a means to boost the energy and C3 of an escape trajectory. Trajectories with two lunar gravity assists are considered and analyzed. Two approaches are applied and tested for the design of missions aimed at Near-Earth asteroids. In the first method, indirect optimization of the heliocentric leg is combined to an approximate analytical treatment of the geocentric phase for short escape trajectories. In the second method, the results of pre-computed maps of escape C3 are employed for the design of longer Sun-perturbed escape sequences combined with direct optimization of the heliocentric leg. Features are compared and suggestions about a combined use of the approaches are presented. The techniques are efficiently applied to the design of a mission to a near-Earth asteroid.
\end{abstract}

Keywords Escape trajectories · Lunar gravity assist $\cdot$ Asteroid missions

\section{Introduction}

For spacecraft interplanetary missions, lunar flybys are often beneficial because they may significantly increase the hyperbolic escape energy $\left(\mathrm{C} 3\right.$, in $\left.\mathrm{km}^{2} / \mathrm{s}^{2}\right)$ for a modest increase in flight time, [1] thus improving the useful mass for a mission to a specific target. This strategy has been used in the past both for missions with low positive values of C3 (STEREO) and larger C3 values (ISEE-3, Nozomi).

Exploration of the solar system requires relatively large values of escape energy. The escape mass that a given launcher provides with a direct launch is a decreasing function of the energy. However, the escape velocity can be properly directed to

Lorenzo Casalino

lorenzo.casalino@polito.it

Gregory Lantoine

gregory.lantoine@jpl.nasa.gov

1 DIMEAS, Politecnico di Torino, Corso Duca degli Abruzzi 24, 10129 Torino, Italy

2 Mission Design and Navigation Section, Jet Propulsion Laboratory, California Institute of Technology, 4800 Oak Grove Dr., Pasadena, CA 91109, USA 
reduce the propellant consumption of the heliocentric flight, so an optimal trade-off usually exists. When the heliocentric flight employs a propulsion system with larger specific impulse compared to the launcher (e.g., electric propulsion), an interplanetary transfer to a near Earth target typically requires escape $\mathrm{C} 3$ of a few $\mathrm{km}^{2} / \mathrm{s}^{2}$. Lunar-gravity-assist (LGA) trajectories may provide a free increase of the escape C3, and the design of LGA escape trajectories for interplanetary missions is the object of the present paper. Most of the literature has focused on low energy trajectories, and only a limited number of papers can be found on high energy escape/capture trajectories [2, 3], with the addition of propulsive maneuvers [4] or with the aid of gravity assists from multiple satellites in the Jovian system [5].

The patched conic approximation is usually adopted for preliminary analysis of interplanetary transfers. The escape phase is therefore treated separately from the heliocentric leg. The purpose of the present analysis to find a suitable escape sequence to start a heliocentric trajectory that reaches the target while maximizing the final mass. The escape sequence is defined, for instance, by date and C3 at departure and following LGA trajectories; the asteroid 2008 EV5 is the target in this paper. It is clear that the methods to analyze the two phases should somehow be complementary for an efficient optimization.

Two approaches can in fact be envisaged to deal with the design on LGA escape trajectories. The first one $[1,6,7]$ pre-computes and maps the escape $\mathrm{C} 3$ as a function of date (i.e., position of the Moon along its orbit) and C3 value before the flyby, and eventually couples these results with the analysis of the interplanetary leg. This approach is necessary when solar perturbation is relevant, as the required numerical integration of the trajectories makes the availability of a pre-computed database of solutions extremely useful and time-saving.

For short escape sequences, when the Sun's gravity is negligible, an analytical treatment of the LGA escape is available. If the optimization of the heliocentric leg is sufficiently fast, an alternative approach [8] may be adopted: the interplanetary leg is treated first and trajectories to the target asteroid are computed for different departure dates and values of hyperbolic excess velocity. For each trajectory, feasibility and performance of a LGA escape are then computed by means of the analytical approximation, thus providing an immediate evaluation of the mission overall performance. In addition, the approximate solution may also provide tentative solutions for a more detailed analysis.

In this paper the two approaches are applied and tested for the design of trajectories aimed at Near-Earth asteroids, with 2008 EV5 being the reference target; solar electric propulsion (SEP) is employed. Escape trajectories with two lunar gravity assists are considered. First, short trajectories, which should be less affected by solar perturbation, are treated with the approximate analytical approach coupled with an indirect optimization method developed at Politecnico di Torino. This approach directly provides evaluation/optimization of launch $\mathrm{C} 3$ and mass, escape C3 and final mass at target arrival. Solar perturbation is relevant during longer Moon-to-Moon transfers and it is introduced for the evaluation of the pre-computed C3 values that correspond to planar escape sequences and assess their feasibility. These results are then coupled with direct optimization of the interplanetary leg, performed with JPL's tool MALTO 
[9], for a complete mission optimization. The features of the two approaches are presented.

\section{Analytical Approach for Unperturbed Escape}

In preliminary analysis, the patched-conic approximation is commonly adopted and the two-body problem equations are used to describe the motion of the point-mass spacecraft (with variable mass). For this approach, the heliocentric phase is treated with an indirect optimization method, based on the theory of optimal control. $[10,11]$

The indirect optimization method assumes escape date, mass and hyperbolic excess velocity magnitude as initially assigned. Escape date and velocity magnitude are parametrically varied to obtain the corresponding set of escape velocity components and target arrival masses for an escape mass initially fixed at $10000 \mathrm{~kg}$. A 28-day window is sampled with a 1-day step to consider a whole lunar period. The initial escape mass will then be updated after the escape analysis and the corresponding final mass at arrival will thus be evaluated.

The optimization procedure provides the escape velocity components, the optimized arrival date and the control time-history (thrust magnitude and direction) to maximize the final mass. The procedure takes advantage of the limited computational effort required by the indirect method, as each trajectory is optimized in less than 1 second on a standard 64-bit Intel i7 3.6 GHz processor.

For each escape condition, an approximate analysis is carried out to define the feasibility of LGA escape trajectories, compute the actual escape mass and re-evaluate the corresponding mass at target arrival. The best solution, that is the one that maximizes the arrival mass, is then selected. Due to the LGA feasibility constraint, the departure date will generally be different from the optimal escape date of the heliocentric leg. This combination of indirect heliocentric optimization and analytical analysis of the flyby was used in Ref. [8] for a single lunar flyby, and is here extended to trajectories with a double lunar flyby.

The LGA trajectory must reach given hyperbolic escape conditions determined with the above mentioned heliocentric analysis. Escape time and velocity (relative to the Earth) components in the J2000 heliocentric ecliptic frame are specified. The time of the last flyby is assumed to coincide with the start of the heliocentric leg (which assumes coincident positions for Earth and spacecraft). Variables are made non-dimensional by using the Earth equatorial radius and the corresponding circular velocity as reference values. Position and velocity of the Moon at escape time are obtained from JPL Ephemerides ${ }^{1}$ DE405. The osculating orbit is employed for Keplerian propagation of the Moon's motion. The approximate analysis is carried out with a reference frame based on the Moon's osculating orbit: $x$-axis towards the ascending node of the Moon's orbit with respect to Earth's equator, z-axis along angular momentum, y-axis to complete a right-handed reference frame: $\boldsymbol{V}_{\text {esc }}$ is the escape velocity vector expressed in this frame.

\footnotetext{
${ }^{1}$ JPL Planetary and Lunar Ephemerides, https://ssd.jpl.nasa.gov/?planet_eph_export, accessed April 3, 2017. 
The analysis is based on a patched-conic approximation that neglects the dimension of the Moon's sphere of influence. The trajectory is split into three geocentric legs. The inner leg (subscript 1) goes from trajectory perigee (usually imposed by the launcher) to the Moon; the intermediate leg (subscript 2) is a Moon-to-Moon transfer, the outer leg (subscript 3) goes from the Moon to escape. LGA is modeled as an instantaneous relative velocity rotation at the Moon's intercept, which separates the geocentric legs. The trajectory is analyzed backward.

\section{Moon to Escape}

This analysis follows the work of Okutsu et al. [12] and is here summarized. The Moon's orbit is on the $\mathrm{x}-\mathrm{y}$ reference plane and its angular position at the last flyby is known. This position must coincide with one of the nodes of the spacecraft escape hyperbola, so that two values are possible for the right ascension of the ascending node (RAAN) $\Omega_{3}$. For each value of $\Omega_{3}$, the remaining orbital parameters of the escape leg hyperbola can, in fact, be determined.

The escape velocity gives

$$
a_{3}=-\frac{1}{V_{e s c}^{2}}
$$

A unit vector pointing to the ascending node $\boldsymbol{u}_{n}$ (components along $\mathrm{x}, \mathrm{y}$ and $\mathrm{z}$ are $\cos \Omega_{3}, \sin \Omega_{3}$, and 0 , respectively) defines the remaining orbital elements: first, the angle between ascending node and escape velocity $\alpha=\cos ^{-1}\left(\boldsymbol{u}_{n} \cdot \boldsymbol{V}_{\text {esc }} / V_{\text {esc }}\right)$ is determined. Then, the unit vector along angular momentum $\boldsymbol{u}_{h}$ is computed; it results to be parallel and concurrent with $\left(\boldsymbol{u}_{n} \times \boldsymbol{V}_{\text {esc }}\right)$ when the escape velocity component along the z-axis $V_{e s c, z}$ is positive, whereas it is in the opposite direction when $V_{e s c, z}$ is negative. The inclination is thus related to the angular momentum component along the $\mathrm{z}$-axis

$$
i_{3}=\cos ^{-1}\left(u_{h, z}\right)
$$

Since the flyby is at a node, one has (see for example Fig. 1; other geometries [12] may be found but the analysis does not present relevant differences) either $\alpha+\Phi-$ $\omega_{3}=\pi$ (positive $V_{e s c, z}$, an index $i_{z}=+1$ is introduced) or $\alpha-\Phi+\omega_{3}=\pi$ (negative $V_{e s c, z}$, the index is $\left.i_{z}=-1\right)$, where the hyperbola half-angle $\Phi=\cos ^{-1}\left(1 / e_{3}\right)$, i.e., the difference between $\pi$ and the true anomaly at infinity) has been introduced. The distance from the Earth must be the same for the spacecraft and the Moon at flyby, that is,

$$
r_{M}=\frac{a_{3}\left(1-e_{3}^{2}\right)}{1+e_{3} \cos v_{3}}
$$

In general, flyby can occur at either node for any $V_{e s c, z}$, with the true anomaly at flyby $v_{3}=-\omega_{3}$ (flyby at ascending node, an index $i_{a}=+1$ is introduced) or $\nu_{3}=-\omega_{3}+\pi$ (descending node, $i_{a}=-1$ ). However, flyby at descending node for positive $V_{e s c, z}$ and at ascending node for negative $V_{e s c, z}$ cannot take place if $\nu_{3}<-\Phi$ (the node would be on the wrong branch of the hyperbola).

By manipulating these equations one gets

$$
i_{a} i_{z} \sin \alpha \sqrt{e_{3}^{2}-1}=-\left(1-i_{a} \cos \alpha\right)-\left(a_{3} / r_{M}\right)\left(e_{3}^{2}-1\right)
$$




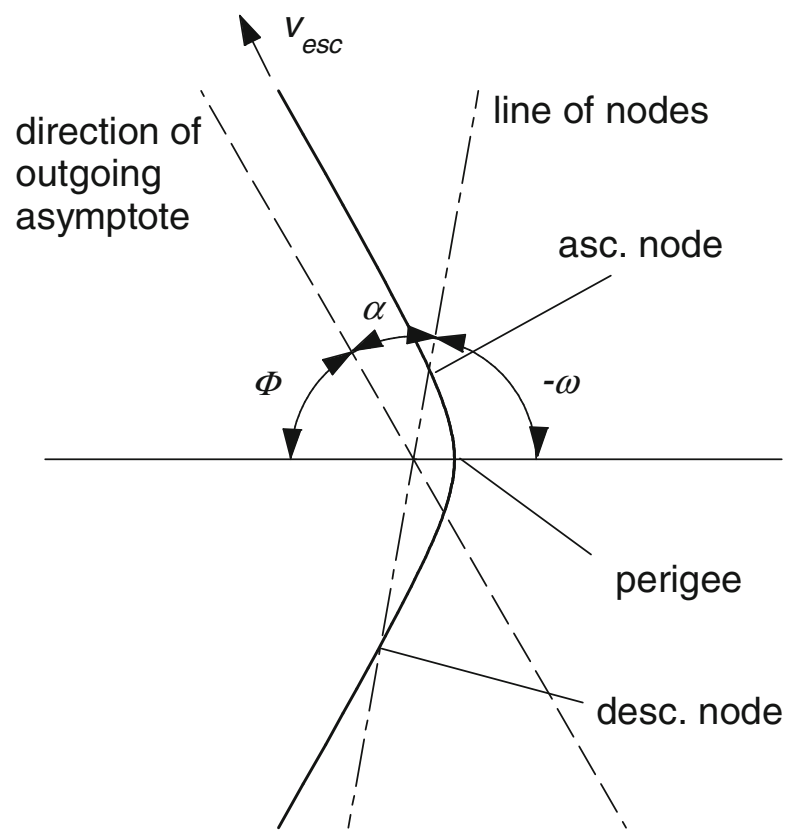

Fig. 1 Geometry of generic escape hyperbola after last flyby for positive $\boldsymbol{V}_{\boldsymbol{e s c}, \boldsymbol{z}}$

which is squared to obtain a quadratic equation in $e_{3}^{2}-1$. The quadratic equation is solved to obtain $e_{3}$. The largest of the two solutions (plus sign in the quadratic solving formula) is the only admissible solution of the radical (4) for flyby at ascending node and positive $V_{e s c, z}$ or flyby at descending node and negative $V_{e s c, z}$. The lower solution (minus sign) must instead be selected when flyby and escape are on opposite sides with respect to the direction of the outgoing asymptote. This solution does not exist when the hyperbola crosses the reference plane only once, that is, when $\nu_{3}<$ $-\Phi$. Once $e_{3}$ has been determined, $\Phi, \omega_{3}$, and $v_{3}$ are immediately obtained. From the orbital elements, one easily finds the relative velocity at Moon's flyby $\boldsymbol{V}_{\infty+}$. For the sake of simplicity, in this approximate analysis the Moon's orbit is assumed to be circular to compute $r_{M}$ and the relative velocity vector.

\section{Moon to Moon}

Three kinds of Moon to Moon transfer (subscript 2) are considered. In resonant transfers, spacecraft and Moon orbital periods are in a ratio of small integer numbers (e.g., 2:1); the Moon is intercepted at the same place after an integer number of revolutions. In backflip transfers the Moon is intercepted at points 180 degrees apart, that is, at the intersections of the spacecraft and the Moon orbit planes. In planar transfers, the trajectory lies entirely on the Moon's orbit plane.

For resonant transfers, semimajor axis and velocity magnitude at flyby are known from the selected orbital resonance. Since the hyperbolic excess velocity is conserved 
during a flyby, its value is also known. If the Moon's orbit is assumed to be circular and the velocity components $u, v$, and $w$ in the radial, eastward and northward directions are introduced, one has

$$
V_{\infty-}^{2}=u^{2}+\left(v-V_{M}\right)^{2}+w^{2}=V^{2}-2 v V_{M}=V_{\infty+}^{2}
$$

where the eastward velocity component at $r=r_{M}$ is $v=\sqrt{p_{2}} / r_{M} \cos i_{2}$. This equation becomes Tisserand's equation, rewritten as

$$
\frac{1}{a_{2}}+2 \sqrt{\frac{a_{2}\left(1-e_{2}^{2}\right)}{r_{M}^{3}}} \cos i_{2}=\frac{3}{r_{M}}-V_{\infty}^{2}
$$

which is solved for $e_{2}$ for inclination values varied at 1-degree steps from 0 to 180 degrees. Check on perigee height is performed to eliminate trajectories that would crash on Earth. The remaining orbital parameters are easily determined as in the previous leg. Only 1:1 and 2:1 resonances are considered to avoid orbits that move the spacecraft to large distances from the Earth, where the Sun's perturbation could significantly alter the trajectory.

Backflip transfers see an inclined spacecraft orbit that, at \pm 90 degrees from its perigee, intercepts the Moon orbit. Thus, $p_{2}=r_{M}$ and $v_{f b}= \pm \pi / 2$. The time equation is iteratively solved to find the eccentricity that matches the required timeof-flight. Again, to avoid large distances from the Earth, only intercept of the Moon after 1.5 revolution is considered, with the spacecraft performing either 0.5 or 1.5 revolutions on an elliptic orbit. Finally, in addition, a 1:1 resonant transfer with the spacecraft also on an inclined circular orbit and flybys after $0.5,1$ or 1.5 revolutions is also analyzed. In any case, Eq. 6 provides the inclination.

For planar transfers, the only known orbit parameter is the inclination ( 0 with respect the lunar orbit plane). Four conditions for this leg fix the radius at flybys (equal to the radius of the Moon's orbit: therefore, the flybys are symmetric with respect to the line of apsides) and the constant magnitude of the hyperbolic excess velocity. Time equation must also be fulfilled to achieve lunar encounter. These conditions determine $a_{2}, e_{2}$ and the true anomalies at flybys (and $\omega_{2}$, as a consequence). The nonlinear system is solved numerically, by introducing the ratio of periapsis radius to the Moon's orbit radius $\rho=r_{p} / r_{M}$ as additional unknown. One has

$$
\begin{gathered}
e_{2}=\frac{1-\rho}{\rho-\cos v_{f b}} \\
a_{2}=\frac{\rho}{1-e_{2}} r_{M}
\end{gathered}
$$

and the time of flight is expressed as a function of $\rho$ and $v_{f b}$, and solved numerically for $v_{f b}$, given $\rho$. The latter is then iterated until (6) is satisfied. The leg is characterized by the direction of the radial velocity at flybys, which can be either inbound (' $\mathrm{i}$ ', when negative) or outbound ('o', when positive). Figure 2 illustrates the four possible combinations (i.e. 'oi', 'oo', 'ii', 'io' transfers). Note that the 'ii' and 'oo' families correspond to resonant transfers in the absence of perturbations, but the resonance is lost when Sun's gravity is considered; for simplicity, they are not considered in this method. Limiting to two the number of revolutions, there are therefore four combinations available; two 'io' transfers (the spacecraft performs more than one revolution, 


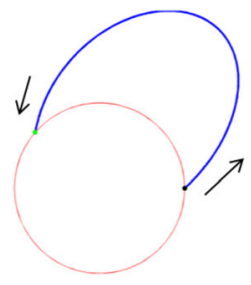

oi

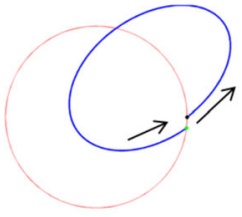

o०

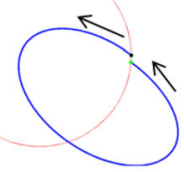

ii

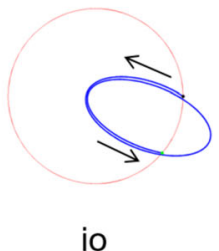

io

Fig. 2 Classification of Moon-to-Moon transfers

the Moon either less or more than one) and two 'oi' transfers (the Moon performs more than one revolution, the spacecraft either less or more than one). Note that the formulation of the time equation takes specific forms according to the transfer under examination.

\section{Perigee to Moon}

Trajectories from perigee to the Moon (subscript 1) that allow feasible flybys to match the escape conditions are here found. Intercept is again on the reference plane and must occur at a node of the spacecraft orbit: $\Omega_{1}=\Omega_{2}$ when the flyby is either at the ascending or at the descending node of both orbits, whereas $\Omega_{1}=\Omega_{2}+\pi$ when it is at the ascending node of one orbit and descending node of the other one.

The magnitude of the relative velocity before and after the flyby must be the same, which gives the radical equation

$$
\left(V_{\infty+}^{2}-3 / r_{M}\right)+\left(1-e_{1}\right) / r_{p}=-2 \sqrt{r_{p} / r_{M}^{3}} \cos i_{1} \sqrt{1+e_{1}}
$$

Among the solutions, the larger one (plus sign) must be selected for $i_{1} \leq \pi / 2$, whereas the correct solution is the lower one (minus sign) for retrograde orbits $i_{1} \geq \pi / 2$. From $e_{1}$, one has $a_{1}=r_{p} /\left(1-e_{1}\right)$. The solution must be discarded for elliptical orbits $\left(a_{1}>0\right)$ if the apogee $r_{a}=a_{1}\left(1+e_{1}\right)$ is lower than $r_{M}$. For acceptable solutions, $r_{M}=a_{1}\left(1-e_{1}^{2}\right) /\left(1+e_{1} \cos v_{1}\right)$ provides $v_{1}$ (only outgoing trajectories are here considered and $\left.0<v_{1} \leq \pi\right)$. The argument of periapsis is then determined, being $v_{1}=-\omega_{1}$ (ascending node) or $v_{1}=-\omega_{1}+\pi$ (descending node).

Velocity rotation at both flybys is given by the angle between the computed $V_{\infty+}$ and $V_{\infty}$ - vectors

$$
\delta=\cos ^{-1}\left[\left(V_{\infty-} \cdot V_{\infty+}\right) / V_{\infty}^{2}\right]
$$

According to the patched-conic approximation

$$
\delta=2 \sin ^{-1} \frac{\mu_{M} / r_{p s}}{V_{\infty}^{2}+\mu_{M} / r_{p s}}
$$

with $\mu_{M}$ being the Moon's gravitational parameter and $r_{p s}$ the flyby periselenium, which can thus be determined. Trajectories are deemed feasible when the periselenium is at least $50 \mathrm{~km}$ above the Moon's surface. 
For any feasible flyby, the values of position and velocity at perigee $\left(V_{p}\right)$ are evaluated and then rotated to the J2000 geocentric frame to determine the corresponding latitude, longitude and azimuth. The departure energy gives the required launch $\mathrm{C} 3$. Azimuth (launch occurs from Kennedy Space Center at 28.5 degrees latitude) and $\Delta V_{p}=V_{p}-V_{c}$ allow one to evaluate the mass that the launcher can insert into the escape trajectory. The following assumptions are here made to replicate the Delta IV Heavy performance. [13] The starting mass on the initial 200-km parking orbit is the sum of useful mass $\left(m_{u}\right)$ and upper stage dry mass $m_{d}(3550 \mathrm{~kg})$. The useful mass given by NASA's Launch Vehicle Performance Website ${ }^{2}$ is here approximated with the quadratic equation

$$
m_{u}=26280-.6642(A-90)^{2}
$$

where $m_{u}$ is in $\mathrm{kg}$ and the azimuth $A$ in degrees. The following burns sum up to $\Delta V=1.046\left(V_{p}-V_{c}\right)$, that is the difference between the perigee velocity at the start of the trajectory to the Moon and the circular velocity on the parking orbit, with the addition of a $4.6 \%$ margin, which has been selected to attain a reference value of $9995 \mathrm{~kg}$ for the escape mass when $\mathrm{C} 3=-1.5 \mathrm{~km}^{2} / \mathrm{s}^{2}$. The useful escape mass is evaluated with the rocket equation

$$
m_{e s c}=\left(m_{u}+m_{d}\right) \exp [-\Delta V / c]-m_{d}-m_{P A / P A F}
$$

where the stage dry mass and the payload adapter/payload attach fitting mass (900 $\mathrm{kg}$ ) are subtracted from the final mass. The stage effective exhaust velocity $c$ corresponds to a $460 \mathrm{~s}$ specific impulse. The heliocentric leg is then re-optimized with the correct escape mass for each date that allows for a feasible LGA sequence (and provides a reasonable mass).

\section{Numerical Approach for Solar-Perturbed Escape}

For long lunar-assisted escape sequences, solar gravitational perturbations between the two lunar flybys may significantly alter the trajectory and naturally produce additional escape energy. [1] However, designing solar-perturbed trajectories connecting two lunar flybys is challenging using the method described in the last section because these trajectories are no longer simply conic.

Switching to another approach, a pre-computed database of Moon-to-Moon transfers in the Sun-Earth CRTBP is used to connect two lunar flybys. Moon gravity is neglected in this analysis and trajectories depart from and arrive at the Moon's center. Planar trajectories on the Moon's orbital plane are considered. This database is described in detail in Ref. [7]. Moon-to-Moon transfers are grouped in different families according to the approximate number of lunar revolutions between lunar encounters (a classification analogous to traditional resonances in the two-body problem). As a result, each family name starts with an uppercase letter whose order in the

\footnotetext{
${ }^{2}$ Launch Vehicle Performance Website, https://elvperf.ksc.nasa.gov/pages/Query.aspx, accessed April 3, 2017.
} 
alphabet corresponds to the approximate number of months between lunar encounters. In addition, two lowercase letters, "o" and "i", are added after the uppercase letter to specify whether the first and second lunar flybys are outbound or inbound, respectively.

The families are parameterized by the initial lunar relative velocity and the initial solar phase angle (angle between Sun-Earth and Earth-Moon lines at the first lunar flyby). Note that each trajectory within a family corresponds to a different solar phase angle. In the context of this paper, an initial lunar relative velocity (i.e., hyperbolic excess velocity) of $1 \mathrm{~km} / \mathrm{s}$ is appropriate. In fact, direct launch trajectories to the Moon tend to produce similar lunar relative velocity values when encountering the Moon for the launch C3 values of interest in this application (between -2 and 0 $\mathrm{km}^{2} / \mathrm{s}^{2}$ ). Only families with $1 \mathrm{~km} / \mathrm{s}$ initial lunar relative velocity will be therefore considered in this section. The exact value is not critical because of the low sensitivity of the families with respect to initial lunar relative velocity. [7] Complete "oi" and "ii" families of solar-perturbed Moon-to-Moon transfers with initial relative velocity of $1 \mathrm{~km} / \mathrm{s}$ are shown in Fig. 3. One can see that the trajectories are strongly affected by variations in solar phase angles. Contrary to the analytical approach, the "ii" families are considered here for completeness, however the results would not change significantly if they were excluded.

Now that all possible trajectories connecting two lunar flybys have been identified, the next step is to enumerate all possible escape options and characterize how the maximum achievable escape C3 varies with departure direction. For all "oi" and "ii" family members the second lunar flyby is modeled as an instantaneous rotation of the hyperbolic excess velocity. The bending angle between the incoming and outgoing $\boldsymbol{v}_{\infty}$ vectors at the final lunar flyby is sampled at $0.1 \mathrm{deg}$ increments, in order to span the complete range of possible escape directions and energies, assuming Keplerian motion after the flyby and circular Moon's orbit. A minimum flyby altitude of $50 \mathrm{~km}$ is enforced, which sets an upper bound in the achievable bending angles. Then the resulting escape $\mathrm{C} 3$, right ascension and declination values of the escape asymptote are recorded for each sampled flyby condition and stored in the lunar escape database. Note that the declination is measured with respect to the ecliptic plane since the Moon is assumed to be in the same plane as the Sun and the Earth in the simplified model of the Moon-to-Moon transfer database. [7] To simplify the search space, "oo" and "io" families, as well as lunar backflips, are not included in this analysis (adding these families in the analysis is a possible future work topic). In addition, families with transfer durations longer than 6 months are not included either to avoid unreasonable flight times.

For all considered "oi" and "ii" families, Fig. 4 shows the maximum achievable escape C3 versus the pump angle of the outbound asymptote (see Fig. 5) with respect to the Earth velocity vector, where an escape pump angle of 0 deg denotes a near-Hohmann transfer to NEOs. All results in Fig. 4 are for planar escapes (0-deg declination). The $\mathrm{C} 3$ data of all families are then combined in Fig. 6 to produce one single max C3 vs pump angle curve. Note that the curve would be probably smoother if longer families had been considered (such as the $\mathrm{G}$ and $\mathrm{H}$ families, see Ref. [7]). As shown in Fig. 6, solar-perturbed double lunar flyby sequences can produce an escape $\mathrm{C} 3$ between 2.5 and $3.2 \mathrm{~km}^{2} / \mathrm{s}^{2}$ for planar departures. Large C3 values higher than 3 


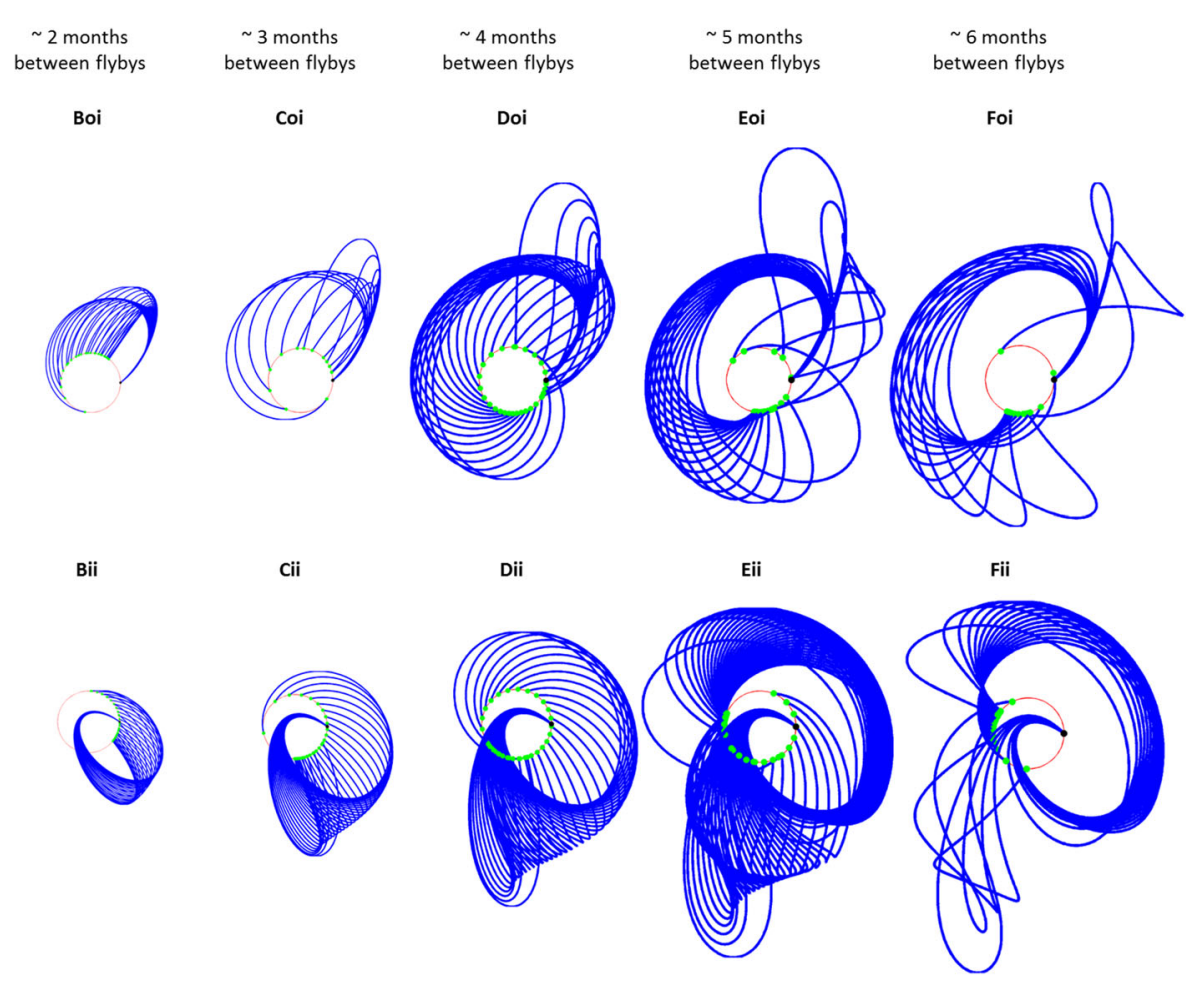

Fig. 3 Complete families of solar-perturbed Moon-to-Moon transfers for an initial lunar relative velocity of $1 \mathrm{~km} / \mathrm{s}$. The Moon's positions at the initial and final flybys are shown by black and green dots, respectively. Within a given family, each trajectory corresponds to a different solar phase angle. To visually emphasize variations between family members, all trajectories are rotated to start at the same lunar location on the $\mathrm{x}$-axis

$\mathrm{km}^{2} / \mathrm{s}^{2}$ are available for a narrow range of pump angles between 120 and $130 \mathrm{deg}$. However, to be conservative, only the lowest $\mathrm{C} 3$ value is kept across all pump angles (flat dashed line in Fig. 6). In other words, it is assumed that the maximum achievable $\mathrm{C} 3$ for a planar lunar escape is $2.5 \mathrm{~km}^{2} / \mathrm{s}^{2}$ for all right ascension directions. This conservatism is necessary because of the simplifications in the model adopted in the database (particularly the circular orbit of the Moon in the ecliptic plane). It is worth noting that this method only finds a lower limit for escape $\mathrm{C} 3$ but does not provide its actual value and its relation to the launch $\mathrm{C} 3$.

The procedure described above is repeated for increasing declination angles up to $85 \mathrm{deg}$. Figure 7 shows how the resulting available escape $\mathrm{C} 3$ varies with declination. This curve gives the maximum escape capability a double lunar flyby is guaranteed to provide (at least in the simplified CRTBP model of the database). As expected, the maximum escape $\mathrm{C} 3$ decreases with increasing declination, since a flyby is most effective when the $\boldsymbol{v}_{\infty}$ vector of the spacecraft aligns with the orbital velocity of the encountered body. Nevertheless, the escape $\mathrm{C} 3$ tapers off in a more gradual way compared to the analytic approach for unperturbed escape and other previous results 


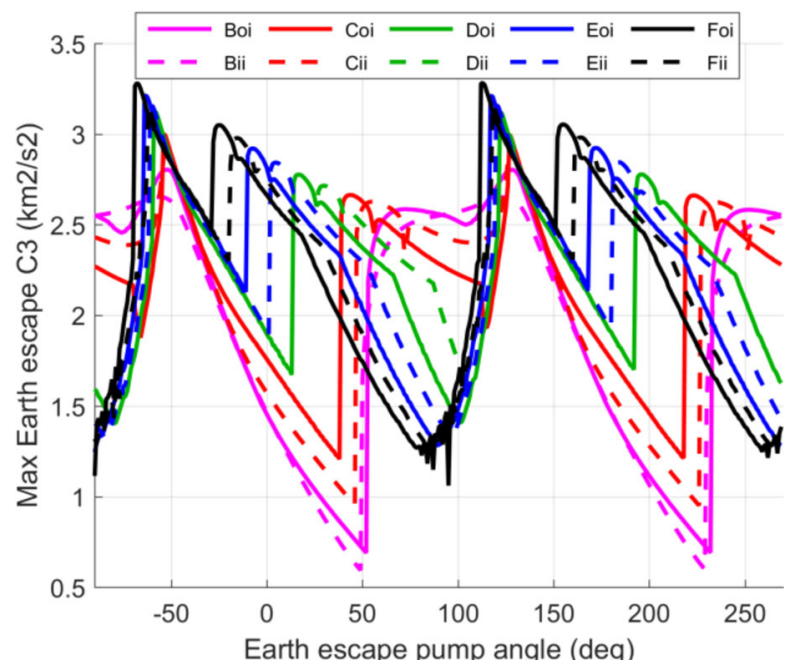

Fig. 4 Maximum escape C3 vs pump angle for each solar-perturbed double lunar flyby family (0-deg declination)

in the literature, which used two-body approximation only and similar initial relative velocity with the Moon. [14] An escape C3 up to $1.5 \mathrm{~km}^{2} / \mathrm{s}^{2}$ is guaranteed for any right ascension and declination directions. Note that these results are also applicable for the reverse problem of capturing a spacecraft in the Earth-Moon system from deep space. The maximum declination available with an escape, or arrival (for Earth capture problems), C3 of $2 \mathrm{~km}^{2} / \mathrm{s}^{2}$ is $+/-30 \mathrm{deg}$. These values are consistent with the typical C3 and declination constraints used in the literature. [15]

To facilitate the design of the interplanetary trajectory when a lunar escape sequence is exploited, the curve shown in Fig. 7 may be viewed as a "lunar-assisted" launch vehicle curve. In fact, the performance of a given launch vehicle is effectively boosted by the lunar escape technique. Since the nominal escape sequence is ballistic, the curve presented in Fig. 7 can be applied to any missions. Ideally, the entire curve should be provided to an interplanetary trajectory optimization tool so that it is possible to move along that curve during the optimization process and find the optimal solution with best escape conditions. The adaptability of the approach to indirect

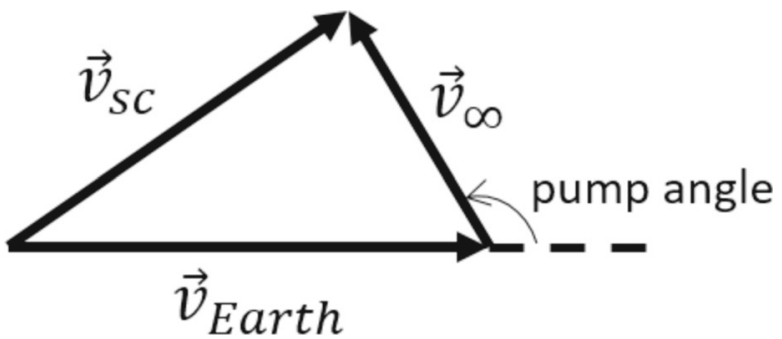

Fig. 5 Pump angle definition diagram 


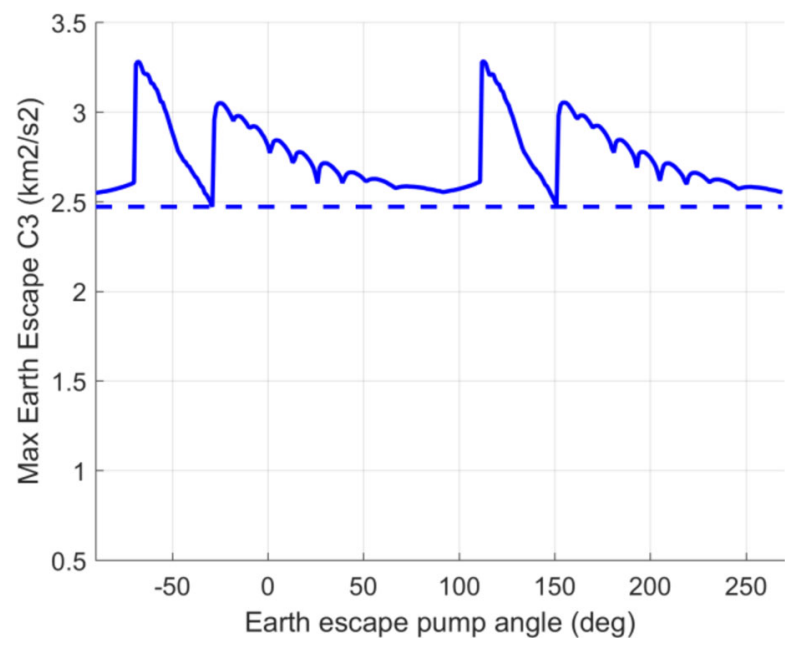

Fig. 6 Combined maximum escape C3 vs pump angle (0-deg declination). The flat dashed line is the conservative $\mathrm{C} 3$ value selected for this particular declination

methods will be object of future work. In practice, however, it is often not possible to enter directly the full curve in the existing tools; in that situation, one can simply run multiple cases with different escape C3 and declination constraints along that curve and pick the best trajectory among all cases. In this analysis, the MALTO software performs this first interplanetary optimization. MALTO is a preliminary low-thrust trajectory design tool that uses a series of impulsive burns to simulate continuous low-thrust trajectory arcs about a single gravitational body and a direct optimization scheme. [16] Because of the inherent approximations involved in the lunar-assisted escape curve, it is not necessary to adopt a high-fidelity trajectory design tool.

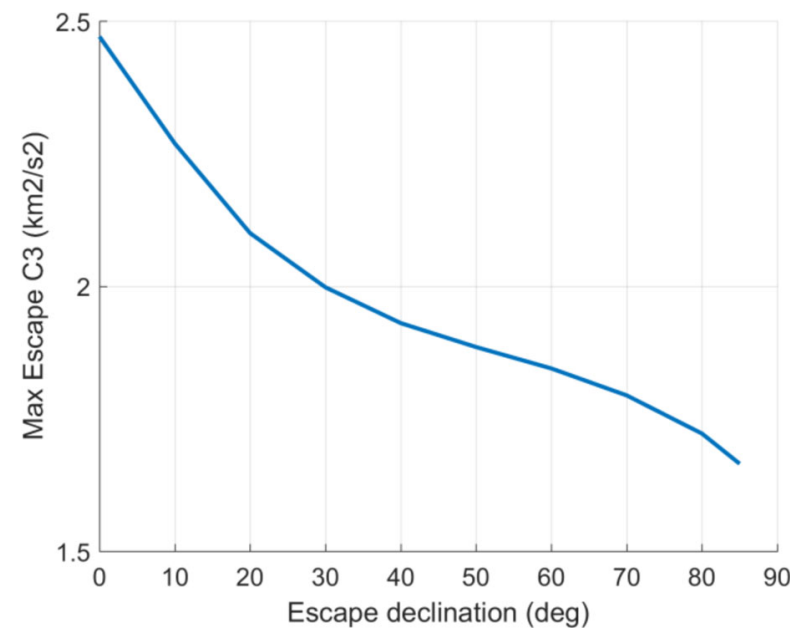

Fig. 7 Maximum escape C3 vs declination with lunar-assisted escape 
Once an optimal interplanetary solution is computed, the corresponding escape conditions are recorded (magnitude and direction of the hyperbolic velocity vector). Then one can simply look up the escape database and retrieve a double lunar flyby sequence that matches these particular escape conditions. This solution is then optimized taking into account the real ephemeris and the full gravity of the Sun, the Earth and the Moon. Typically, the optimized, high-fidelity trajectory closely resembles the initial guess trajectory in the simplified model. [7] Note that the departure date does not generally match exactly the optimal escape date of the interplanetary trajectory because this date is constrained by the phasing of the Moon. The departure date can change by up to 14 days to ensure the Moon is at a favorable location. Fortunately, SEP trajectories tend to offer some flexibility in the launch (or escape) date, so this slight discrepancy is generally not an issue.

The last step is to optimize the trajectory end-to-end (i.e. with lunar escape and interplanetary legs) to reconcile the departure date across all legs, produce a fully continuous trajectory and optimize further the escape conditions using the actual, high-fidelity lunar escape sequence without approximations. A high-fidelity trajectory optimization tool is necessary for this step, such as Mystic [17] or Copernicus. [18]

\section{ARRM Example}

The lunar escape design methodologies are applied to a rendezvous mission with 2008 EV5, which is consistent with the reference ARRM scenario. [19] Launch is constrained to be no earlier than December 2021, which yields an escape date in the mid-2022 range. All other mission assumptions are detailed in Ref. [19].

For the short escape trajectories, the interplanetary leg is first optimized with the indirect method and the optimal departure date for the heliocentric leg (June 18, 2022 ) is determined. Trajectories with escape dates at 1-day steps in a 28 day window centered at the optimal departure are also evaluated to explore a whole lunar period. Values of escape velocities in the $1.3-1.4 \mathrm{~km} / \mathrm{s}$ range are assumed. The optimal escape declination is about 67.5-68 degrees. Escape trajectories with a single LGA are not available. Among trajectories with two LGAs, backflips are feasible both for last flyby at the ascending and descending node of the escape hyperbola and both for elliptic and circular orbits. Elliptic transfers with last flyby at the descending node have slightly better performance and attention is here focused on them. The actual arrival mass is estimated based on the results of the heliocentric leg (propellant consumption for a reference escape mass of $10000 \mathrm{~kg}$ ) and geocentric leg (actual escape mass), assuming the propellant consumption of the heliocentric leg to be proportional to the escape mass. The best solution in terms of arrival mass, which corresponds to escape on June 21, 2022 and escape velocity $1.4 \mathrm{~km} / \mathrm{s}$, is picked. The dependence of the heliocentric leg performance on escape velocity is rather weak; for instance, only $30 \mathrm{~kg}$ of additional propellant are needed for a reduction from $1.4 \mathrm{~km} / \mathrm{s}$ to $1.3 \mathrm{~km} / \mathrm{s}$ ( $2 \%$ of the overall propellant consumption, which is about $1500 \mathrm{~kg}$ ). 
Figure 8 shows different views of the resulting optimal lunar escape sequence. The first lunar flyby occurs on May 4, 2022 with an altitude of $670 \mathrm{~km}$. The second lunar flyby occurs on June 14, 2022 (i.e. 1.5 lunar periods after the first flyby) with an altitude of $109 \mathrm{~km}$. The relative velocity is always $1.66 \mathrm{~km} / \mathrm{s}$. Launch (from perigee) occurs on May 2, 2022 with a launch C3 of $0.02 \mathrm{~km}^{2} / \mathrm{s}^{2}$ for a theoretical escape mass of $9664 \mathrm{~kg}$ (eastward launch from 28.5 degrees). However, in reality, it is necessary to add phasing loops after launch to provide a 21-day launch period, which moves back the actual launch date. These phasing loops are described in detail in Ref. [19] and are omitted in this analysis. Note that in an ideal case with impulsive maneuvers and no perturbations, the total $\Delta \boldsymbol{V}$ is split into two impulses, but the propellant consumption does not change: the first impulse, usually provided by the launcher, inserts the spacecraft into an elliptical orbit with proper period to attain the correct phasing and the second one, performed by the spacecraft, into the orbit to intercept the Moon. However, an escape mass of $9550 \mathrm{~kg}$ is assumed (instead of the nominal value 9664 $\mathrm{kg}$ ), to account for additional Xenon and hydrazine consumption [19]. Following this lunar escape sequence, arrival at 2008EV5 then occurs on August 21, 2023 with a final mass, also considering $75 \mathrm{~kg}$ of additional xenon and hydrazine consumption, [19] of $8186 \mathrm{~kg}$.

The escape trajectory is verified by means of a high-fidelity four-body model in an Earth centered frame. Earth's oblateness (up to 8-th degree), lunisolar gravitational perturbation and solar radiation pressure are considered. JPL's DE405 ephemeris are employed for the positions of the perturbing bodies. In agreement with past experience [8], the analytical solution results to be quite accurate. The theoretical escape

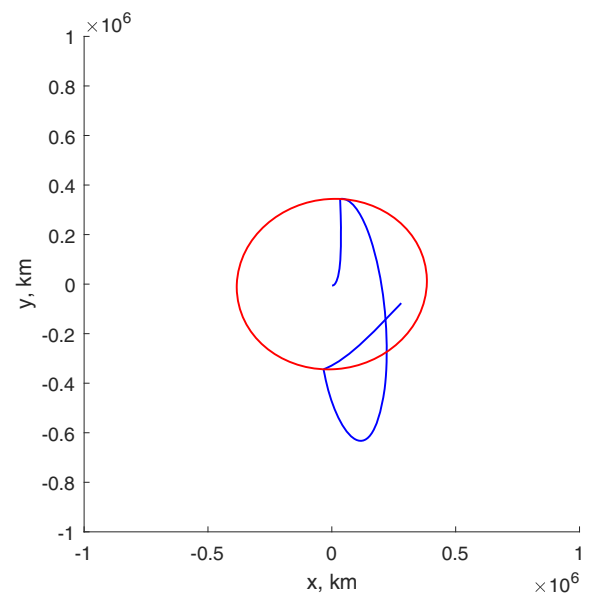

(a) In-plane view.

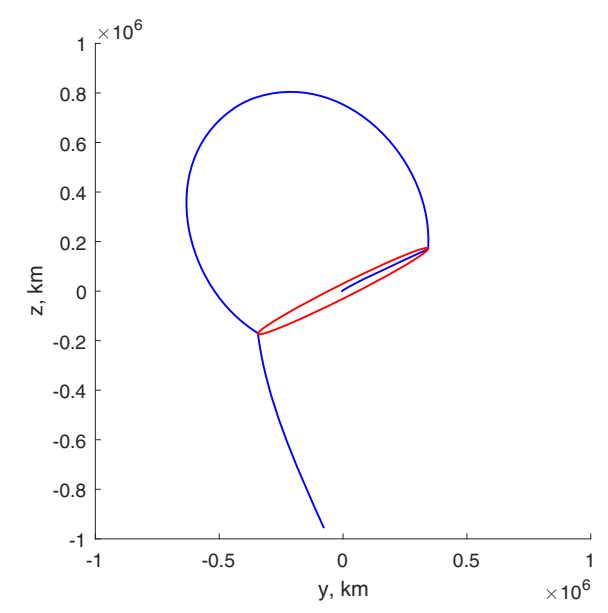

(b) Out-of-plane view.

Fig. 8 Lunar escape trajectory with backflip for the reference ARRM mission concept to 2008EV5 in the equatorial J2000 inertial frame (Earth-centered). Lunar orbit is in red 


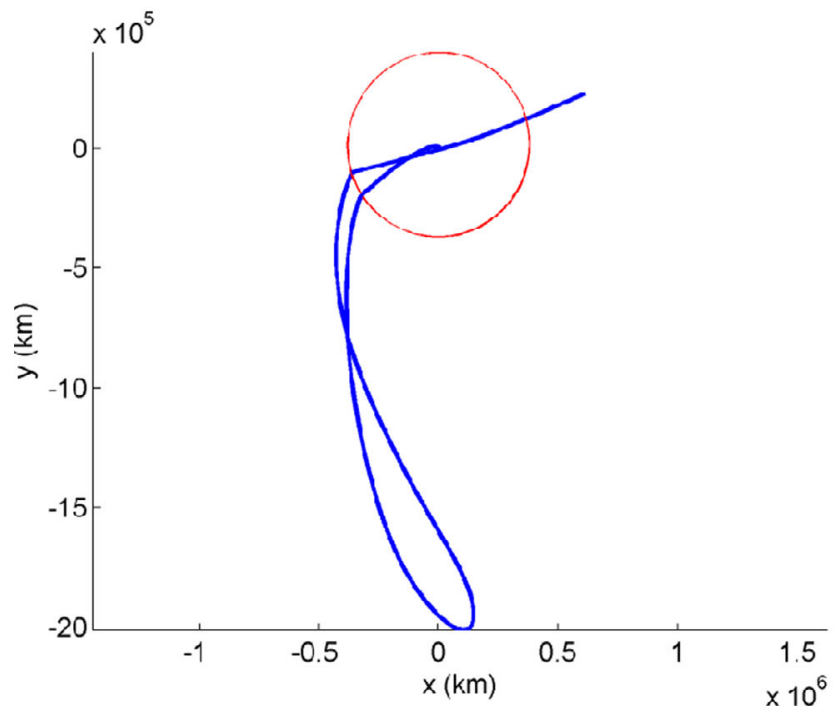

(a) In-plane view.

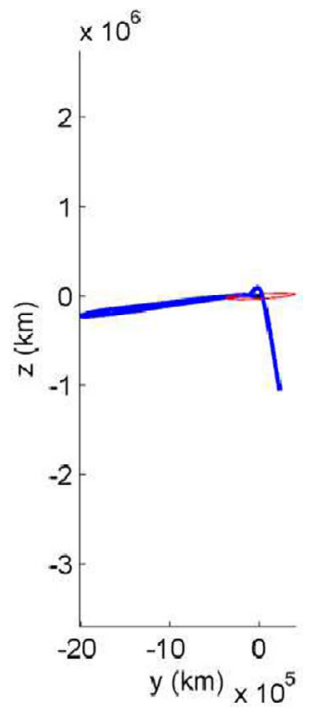

(b) Out-of-plane view.

Fig. 9 Solar-perturbed lunar escape trajectory of the reference ARRM mission concept to 2008EV5 in the ecliptic J2000 inertial frame (Earth-centered). Lunar orbit is in red

mass $(9692 \mathrm{~kg}$ ) is only $28 \mathrm{~kg}$ larger than the value of the approximate solution, as a slightly smaller launch C3 is required, with a mass delivered to the target asteroid of $8210 \mathrm{~kg}$. The only relevant difference concerns the flyby altitudes, which results to be larger in the high-accuracy model.

For the solar-assisted lunar escape design methodology, the interplanetary trajectory is first optimized in Malto using the lunar escape performance curve (see Fig. 7) to find that a June 2022 escape is optimal with -65 deg declination. Looking up the escape database, a member of the Doi family is retrieved that reproduces well the escape conditions. The full trajectory is then optimized in high-fidelity. Figures 9 and 10 show the resulting optimal lunar escape sequence in different frames. The first lunar flyby occurs on February 21, 2022 with an altitude of $5119 \mathrm{~km}$ and a relative velocity of $1.05 \mathrm{~km} / \mathrm{s}$. The second lunar flyby occurs on June 09, 2022 (i.e. 108 days after the first flyby) with an altitude of $55 \mathrm{~km}$ and a relative velocity of $2.15 \mathrm{~km} / \mathrm{s}$. The produced escape $\mathrm{C} 3$ is $1.74 \mathrm{~km}^{2} / \mathrm{s}^{2}$ with an ecliptic declination of $-67.6 \mathrm{deg}$. One can observe that this performance is slightly better than the one advertised in the lunar-assisted escape curve (see Figure 4), which confirms the conservatism embedded in this curve. In this example trajectory, launch occurs on February 18, 2022 with a launch $\mathrm{C} 3$ of $-1.75 \mathrm{~km}^{2} / \mathrm{s}^{2}$. Again, phasing loops must be considered and the actual launch date is moved back to the end of December 2021. The corresponding escape mass is $9821.9 \mathrm{~kg}$ when representative maneuvers during phasing loops are included. Following this lunar escape sequence, arrival at 2008EV5 then occurs on August 25, 2023 with an arrival mass [19] of $8432.8 \mathrm{~kg}$. The improvement with respect to the short escape sequence is about $250 \mathrm{~kg}$. 


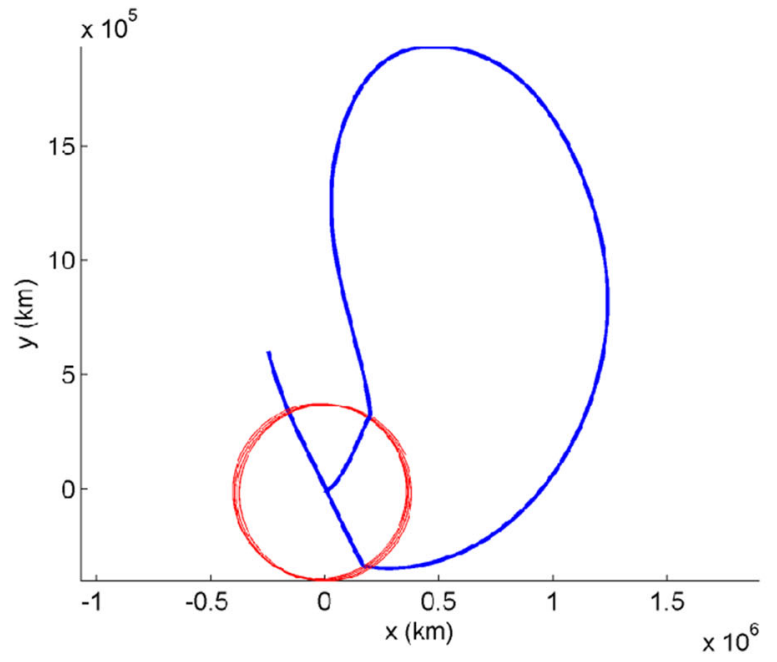

(a) In-plane view.

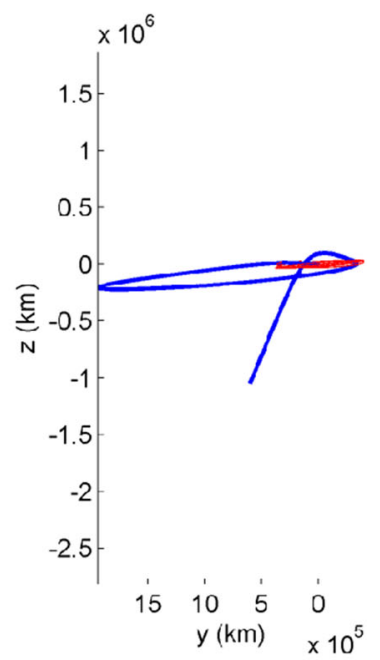

(b) Out-of-plane view.

Fig. 10 Solar-perturbed lunar escape trajectory of the reference ARRM mission concept to 2008EV5 in the Sun-Earth rotating frame (Earth-centered). Lunar orbit is in red

\section{Conclusion}

Methodologies are presented to facilitate the design of double lunar flyby escape sequences suitable for missions to near-Earth asteroids with SEP. The methodologies can however be applied to different missions, provided the required value of escape velocity is not excessively large. A simplified analytic approach deals with short escape sequences which are not decisively affected by solar perturbation. Both planar and noncoplanar Moon-to-Moon trajectories are considered. The numerical example highlights that backflip maneuvers are useful when a large escape declination is sought. Solar-perturbed longer escape sequences are computed by exploiting a pre-computed database to allow mission designers to quickly explore the trajectory space and choose appropriate trajectories for specific missions. Even though the methods assume circular orbit for the Moon, numerical verifications showed that the impact of the eccentricity is limited to a small fraction of the propellant consumption. The representative examples given confirm that these techniques are effective to design missions to NEOs. In particular, adding a lunar escape sequence proved to be critical for designing a feasible trajectory of the ARRM mission concept.

Acknowledgments The research was partially carried out at the Jet Propulsion Laboratory, California Institute of Technology, under a contract with the National Aeronautics and Space Administration, and partially at the Politecnico di Torino on behalf of the Italian Space Agency (ASI) in the framework of the Joint NASA and ASI Feasibility Study on Potential Collaboration in the Asteroid Redirect Robotic Mission.

Funding Information Open access funding provided by Politecnico di Torino within the CRUI-CARE Agreement. 
Open Access This article is licensed under a Creative Commons Attribution 4.0 International License, which permits use, sharing, adaptation, distribution and reproduction in any medium or format, as long as you give appropriate credit to the original author(s) and the source, provide a link to the Creative Commons licence, and indicate if changes were made. The images or other third party material in this article are included in the article's Creative Commons licence, unless indicated otherwise in a credit line to the material. If material is not included in the article's Creative Commons licence and your intended use is not permitted by statutory regulation or exceeds the permitted use, you will need to obtain permission directly from the copyright holder. To view a copy of this licence, visit http://creativecommonshorg/licenses/by/4.0/.

\section{References}

1. McElrath, T.P., Lantoine, G., Landau, D., Grebow, D., Strange, N., Wilson, R., Sims, J.: Using Gravity Assists in the Earth-Moon System as a Gateway to the Solar System, paper GLEX2012.05.5.2x12358, Global Space Exploration Conference (2012)

2. Nock, K., Uphoff, C.: Satellite aided orbit capture, paper AAS 79-165 AAS (1979)

3. Cline, J.K.: Satellite aided capture. Celest. Mech. 19(4), 405-415 (1979). https://doi.org/10.1007/ BF01231017

4. Scott, C., Ozimek, M., Haapala, A., Siddique, F.E., Buffington, B.B.: Dual Satellite-Aided Planetary Capture with Interplanetary Trajectory Constraints. J. Guid. Control. Dyn. 40, 548-562 (2017). https://doi.org/10.2514/1.G002102

5. Lynam, A., Kloster, K.W., Longuski, J.: Multiple-satellite-aided capture trajectories at Jupiter using the Laplace resonance. Celest. Mech. Dyn. Astron. 109, 59-84 (2011). https://doi.org/10.1007/s105 69-010-9307-1

6. Campagnola, S., Jehn, R., Corral Van Damme, C.: Design of Lunar Gravity Assit for the BEPICOLOMBO Mission to Mercury, paper AAS 04-130 AAS (2004)

7. Lantoine, G., McElrath, T.P., Timothy, P.: Families of Solar-Perturbed Moon-to-Moon Transfers, paper AAS 14-233 AAS (2014)

8. Casalino, L., Filizola, L.: Design of High-Energy Escape Trajectories with Lunar Gravity Assist, paper ISTS-2017-d-023/ISSFD-2017-023, ISTS (2017)

9. Sims, J., Finlayson, P., Rinderle, E., Vavrina, M., Kowalkowski, T.: Implementation of a Low-Thrust Trajectory Optimization Algorithm for Preliminary Design. https://doi.org/10.2514/6.2006-6746

10. Bryson, A.E., Ho, Y.-C.: Applied Optimal Control. Hemisphere, New York (1975). Revised printing

11. Casalino, L., Colasurdo, G., Pastrone, D.: Optimal Low-Thrust Escape Trajectories Using Gravity Assist. J. Guid. Control. Dyn. 22(5), 637-642 (1999). https://doi.org/10.2514/2.4451

12. Okutsu, M., Yam, C., Longuski, J., Strange, N.: Cassini end-of-life escape trajectories to the outer planets, technical reports, Advances in the Astronautical Sciences, vol. 129 (2008)

13. Delta IV launch Services User's Guide, tech. rep., United Launch Alliance (2013)

14. Landau, D., McElrath, T.P., Grebow, D., Strange, N.: Efficient Lunar Gravity Assists for Solar Electric Propulsion Missions, paper AAS 12-165, Advances in the Astronautical Sciences, vol. 143 (2012)

15. Merrill, R., Ou, M., Vavrina, M., Jones, C.A., Englander, J.: Interplanetary Trajectory Design for the Asteroid Robotic Redirect Mission Alternate Approach Trade Study, paper AIAA 2014-4457 AIAA (2014)

16. Sims, J., Finlayson, P., Rinderle, E., Vavrina, M., Kowalkowski, T.: Implementation of a Low-Thrust Trajectory Optimization Algorithm for Preliminary Design, paper AIAA 2006-6746, AIAA (2006)

17. Whiffen, G., Sims, J.: Application of a Novel Optimal Control Algorithm to Low-Thrust Trajectory Optimization, paper AAS 01-209, AAS (2001)

18. Ocampo, C., Senent, J., Williams, J.: Theoretical Foundation of Copernicus: a Unified System for Trajectory Design and Optimization, JSC-CN-20552, 4th International Conference on Astrodynamics Tools and Techniques (2010)

19. McGuire, M.L., Strange, N.J., Burke, L.M., McCarty, S.L., Lantoine, G.B., Qu, M., Shen, H., Smith, D.A., Vavrina, M.A.: Overview of the Mission Design Reference Trajectory for NASA's Asteroid Redirect Robotic Mission (ARRM), paper AAS 17-585, AAS (2017)

Publisher's Note Springer Nature remains neutral with regard to jurisdictional claims in published maps and institutional affiliations. 